\title{
Implementation of Wavelet Based Transform for numerical solutions of Partial differential equations
}

\author{
Ali Al-Fayadh ${ }^{1}$ and Nawar Hazim ${ }^{2}$ \\ Department of Mathematics and Computer Applications, Al-Nahrain University, College of Science, Iraq \\ 1)aalfayadh@yahoo.com 2)nawarbmw73@yahoo.com
}

\begin{abstract}
Nonlinear phenomena play a major role in applied mathematics and engineering. The Burger-Fisher equation which is a mixed hyperbolic-parabolic non-linear partial differential equation occurs in various areas of applied sciences and physical applications, such as modeling of gas dynamics and fluid mechanics. In this paper, Haar wavelet method was implemented efficiently in finding the numerical solution of Burger-Fisher equation. This method shows rather rapid convergence than other existing methods. Illustrative examples suggest that using wavelet based method providing a powerful approach to find numerical solutions of BurgerFisher equation. The comparison of numerical results with the exact solutions, and the solutions obtained using some traditional methods such as variational iteration method (VIM) show that the suggested scheme is fairly accurate and viable for solving such problems.
\end{abstract}

Keywords: Burger's-Fisher equation, Haar wavelet method, Variational iteration method.

\section{Introduction}

Nonlinear partial differential equations (NPDEs) were resulted by mathematical modeling of many physical systems and appeared in various fields of engineering and science. The numerical solutions of NPDEs, such as the Burgers-Fisher equation, have attracted much attention due to their potential applications in various fields of fluid dynamics, heat conduction, gas dynamic, traffic flow, applied mathematics and some other fields of science [1-5]. The Burgers-Fisher equation which describes the interaction between the reaction mechanism, convection effect, and diffusion transport [6] is considered in this paper. Many numerical schemes have been proposed for obtaining approximate solutions of the Burger-fisher equation [7-12].

The wavelet series which is shown to be an efficient and accurate method to obtain the approximated solutions of the partial differential equations (PDEs) has been used over the past two decades. This method makes use of two parameters which are the dilation and the translation. The dilation parameter is used for compression of wavelet series solution and the translation parameter is used for the location of wavelet series

solution in $\mathrm{L}^{2}(\mathrm{R})$ space. The method is capable of giving approximated solution to the PDEs in both of the regular and irregular domain. Furthermore, the wavelet function is symmetrical, detects the discontinuities in the equation, and yields solution which is powerful against noise. In addition, the wavelet series solution of a PDE converges faster than the conventional methods of approximation on the basis that the wavelet function has the compact support property. On the other hand, due to the multiresolution analysis property in wavelet domain, it may obtain both time and frequency information which is not feasible with other methods of approximating PDEs.

In this work, the goal is to obtain the approximate solution of the Burger-Fisher equation by using the Haar wavelet method [13-14] and to compare the obtaining results with the exact solution and the iterative methods [15].

Let us consider the Burger-Fisher equation [10] is as follows:-

$u_{t}-u_{x x}+\alpha u^{\gamma} u_{x}+\beta u\left(u^{\gamma}-1\right)=0, \quad 0 \leq x \leq 1, \quad t \geq 0$

with the initial condition given by:

$u(x, 0)=\left(\frac{1}{2}-\frac{1}{2} \tanh \left(\frac{\alpha \gamma}{2(\gamma+1)} x\right)\right)^{\frac{1}{\gamma}}$

And the boundary conditions:

$$
\left.\begin{array}{c}
u(0, t)=\left(\frac{1}{2}+\frac{1}{2} \tanh \left(\frac{\alpha \gamma}{2(\gamma+1)}\left(\frac{\alpha}{\gamma+1}+\frac{\beta(\gamma+1)}{\alpha}\right) t\right)\right) \frac{1}{\gamma}, \quad \mathrm{t} \geq 0 \\
u(1, t)=\left(\frac{1}{2}+\frac{1}{2} \tanh \left[\frac{-\alpha \gamma}{2(\gamma+1)}\left(1-\left(\frac{\alpha}{\gamma+1}+\frac{\beta(\gamma+1)}{\alpha}\right) t\right)\right]\right)^{\frac{1}{\gamma}}, \mathrm{t} \geq 0
\end{array}\right\} .
$$

Where $\alpha, \beta \geq 0$ and $\gamma>0$ are given constants. If $\gamma=1$, Eq.(1) is called the Burger-Fisher equation. When $\alpha$ $=0, \gamma=1$, Eq.(1) is reduced to the Huxley equation which describes nerve pulse propagation in nerve fibre and 
wall motion in liquid crystals [16]. Generalized Burger equation will be obtained when $\beta=0$. This equation when $\beta=0$, has been used to investigate sound waves in a viscous medium by Lighthill [17]. However, it was originally introduced by Burgers [18] to model one-dimensional turbulence.

The boundary conditions (3) are taken from the exact solution which is given by [8-10]

$$
u(x, t)=\left(\frac{1}{2}+\frac{1}{2} \tanh \left(\frac{-\alpha \gamma}{2(\gamma+1)}\left(x-\left(\frac{\alpha}{\gamma+1}+\frac{\beta(\gamma+1)}{\alpha}\right) t\right)\right)\right)^{\frac{1}{\gamma}}
$$

\section{Haar wavelet method}

The wavelet transform or wavelet analysis is a recently developed mathematical tool for many problems. One of the popular families of wavelet is Haar wavelets [19]. In fact the Haar wavelet is the Daubechies wavelet of order 1. Due to its simplicity, the Haar wavelet had become an effective tool for solving many problems arising in many branches of sciences. It has been used since 1910 where the Hungarian mathematician Alfred Haar discovered it. The Haar wavelet is an orthonormal wavelet with compact support. Haar showed that certain square wave function could be translated and scaled to create a basis set that span $\mathrm{L}^{2}(\mathrm{R})$. If we choose scaling function to have compact support over $0 \leq x \leq 1$, that is the Haar wavelet family for $\mathrm{x} \in[0,1]$ is defined as

$$
h_{i}(x)=\left\{\begin{array}{rc}
1 & x \in\left[\xi_{1}, \xi_{2}\right) \\
-1 & x \in\left[\xi_{2}, \xi_{3}\right] \\
0 & \text { otherwise }
\end{array}\right.
$$

Where

$$
\xi_{1}=\frac{k}{m}, \quad \xi_{2}=\frac{k+0.5}{m}, \xi_{3}=\frac{k+1}{m}
$$

where $m=2^{j}, j=0,1, \ldots, J$ indicates the level of the wavelet and the integer $k=0,1, \ldots, m-1$ is the translation parameter. The maximal level of resolution is $J$. The index $i$ in Eq. (5) is calculated from the formula $i=m+k+1$. In the case of minimal values $\quad m=1, k=0$, we have $i=2$. The maximum value of $i$ is $i=2 M=$ $2^{J+1}$. For $i=1$. The function $h_{1}(x)$ is the scaling function for the family of the Haar wavelets which is defined as.

$$
h_{1}(\mathrm{x})=\left\{\begin{array}{cc}
1 & x \in\left[\xi_{1}, \xi_{2}\right) \\
0 & \text { otherwise }
\end{array}\right.
$$

The following integrals are useful for solving second order partial differential equations.

$$
\begin{aligned}
& p_{i}(x)=\int_{0}^{x} h_{i}(x) d x= \begin{cases}x-\xi_{1} & \text { for } x \in\left[\xi_{1}, \xi_{2}\right) \\
\xi_{3}-x & \text { for } x \in\left[\xi_{2}, \xi_{3}\right) \ldots \ldots \ldots \ldots \ldots(8) \\
0 & \text { otherwise }\end{cases} \\
& q_{i}(x)=\int_{0}^{x} p_{i}(x) d x= \begin{cases}\frac{1}{2}\left(x-\xi_{1}\right)^{2} & \text { for } x \in\left[\xi_{1}, \xi_{2}\right) \\
\frac{1}{4 m^{2}}-\frac{1}{2}\left(\xi_{3}-x\right)^{2} & \text { for } x \in\left[\xi_{2}, \xi_{3}\right) \\
\frac{1}{4 m^{2}} & \text { for } x \in\left[\xi_{3}, 1\right] \\
0 & \text { otherwise }\end{cases}
\end{aligned}
$$

Any function $\mathrm{y}(\mathrm{x})$ which is square inegrable on $[0,1]$ can be expressed in terms of Haar wavelet as follows $y(x)=\sum_{i=1}^{\infty} a(i) h_{i}(x)$.

Here, the expansion $y(x)$ is an infinite series. If $y(x)$ is approximated as pricewise constant in each sub-area, then it will be terminated at finite terms, that is

$y(x)=\sum_{i=1}^{2 M} a(i) h_{i}(x)$.

Where the wavelet coefficients a(i), $\mathrm{i}=1,2, \ldots \ldots, 2 \mathrm{M}$ are to be determined.

Now, consider an initial boundary value problem (IBVP) for the nonhomogeneous Burgers-Fisher equation (1) with the initial and boundary conditions

$u(x, 0)=f(x), 0 \leq x \leq 1$,

$u(0, t)=g_{1}(t), t \geq 0$,

$u(1, t)=g_{2}(t), t \geq 0$

Let us divided the interval $[0, \mathrm{~T}]$ into $\mathrm{N}$ equal parts of length $\Delta t=\frac{T}{N}$ and denote $t_{s}=(\mathrm{s}-1) \Delta t$, 
$\mathrm{s}=1,2, \ldots, \mathrm{N}$. We assume a Haar wavelet solution for equation (1) in the form [20],

$\dot{u}^{\prime \prime}(x, t)=\sum_{i=1}^{2 M} a_{s}(i) h_{i}(x)$,

here the dot and prime denote differentiation with respect to $t$ and $x$ respectively, the row vector $a_{\mathrm{s}}$ is constant in the subinterval $t \in\left[t_{s}, t_{s+1}\right]$. Integrating equation (13) with respect to $t$ in the limits $\left[t_{s}, \mathrm{t}\right]$ then by integrating the resultant equation with respect to $x$ in the limits $[0, x]$, and the resultant equation is again integrating with respect to $\boldsymbol{x}$ in the limits $[0, x]$ and differentiating the resultant equation with respect to $\boldsymbol{t}$, we obtain the following equations respectively.to $t$, we obtain the following equations respectively.

$u^{\prime \prime}(x, t)=\left(t-t_{s}\right) \sum_{i=1}^{2 M} a_{s}(i) h_{i}(x)+u^{\prime \prime}\left(x, t_{s}\right)$,

$u^{\prime}(x, t)=\left(t, t_{s}\right) \sum_{i=1}^{2 M} a_{s}(i) p_{i}(x)+u^{\prime}\left(x, t_{s}\right)-u^{\prime}\left(0, t_{s}\right)+u^{\prime}(0, t)$

$u(x, t)=\left(t, t_{s}\right) \sum_{i=1}^{2 M} a_{s}(i) q_{i}(x)+u\left(x, t_{s}\right)-u(0, t)+x\left[u^{\prime}(0, t)-u^{\prime}(0, t)\right]+u(0, t)$

$\dot{u}(x, t)=\sum_{i=1}^{2 M} a_{s}(i) q_{i}(x)+\dot{u}(0, t)+x \dot{u}^{\prime}(0, t)$.

Using the boundary conditions(10)-(11), we have

$u(0, t s)=g_{1}\left(t_{s}\right), \quad u\left(1, t_{s}\right)=g_{2}\left(t_{s}\right)$

$\dot{u}(0, t)=\dot{g}_{1}(t), \quad \dot{u}(1, t)=\dot{g}_{2}(t)$.

Putting $\mathrm{x}=1$ in equations (16) and (17) and using the conditions in (18)-(19), we obtain

$u^{\prime}(0, t)-u^{\prime}\left(0, t_{s}\right)=-\left(t-t_{s}\right) \sum_{i=1}^{2 M} a_{s}(i) q_{i}(1)+g_{2}(t)-g_{1}(t)-g_{2}\left(t_{s}\right)+g_{1}\left(t_{s}\right)$

$\dot{u}^{\prime}(0, t)=-\sum_{i=1}^{2 M} a_{s}(i) q_{i}(1)+\dot{g}_{2}(t)-\dot{g}_{1}(t)$

The wavelet collocation points are defined as

$$
x_{I}=\frac{I-0.5}{2 M}, I=1,2, \ldots, 2 M .
$$

Substituting equation (18)-(21) in equations (14)-(17) and taking $x \rightarrow x_{I}, t \rightarrow t_{s+1}$, we get

$$
\begin{gathered}
u^{\prime \prime}\left(x_{I}, t_{s+1}\right)=\Delta t \sum_{i=1}^{2 M} a_{s}(i) h_{i}(x)+u^{\prime \prime}\left(x_{I}, t_{s}\right), \ldots \ldots \ldots(25) \\
u^{\prime}\left(x_{I}, t_{s+1}\right)=\Delta t \sum_{i=1}^{2 M} a_{s}(i)\left[p_{i}\left(x_{I}\right)-q_{i}(1)\right]+u^{\prime}\left(x_{I}, t_{s}\right)+g_{2}\left(t_{s+1}\right)-g_{1}\left(t_{s+1}\right)-g_{2}\left(t_{s}\right)+g_{1}\left(t_{s}\right) \\
\ldots\left(x_{I}, t_{s+1}\right)=\Delta t \sum_{i=1}^{2 M} a_{s}(i)\left[q_{i}\left(x_{I}\right)-x_{I} q_{i}(1)\right]+u\left(x_{I}, t_{s}\right)+x_{I}\left[g_{2}\left(t_{s+1}\right)-g_{2}\left(t_{s}\right)\right] \\
\quad+\left(1-x_{I}\right)\left[g_{1}\left(t_{s+1}\right)-g_{1}\left(t_{s}\right)\right], \ldots \ldots \ldots \ldots(27)
\end{gathered}
$$

Using the quasilinearization technique [21] to handle the nonlinearity in equation(1).

Now, we have the following scheme

$\sum_{i=1}^{2 M} a_{s}(i)\left[q_{i}\left(x_{I}\right)-x_{I} q_{i}(1)\right]+\dot{g}_{1}\left(t_{s+1}\right)+x_{I}\left[\dot{g}_{2}\left(t_{s+1}\right)-\dot{g}_{1}\left(t_{s+1}\right)\right]-u^{\prime \prime}\left(x_{I}, t_{s+1}\right) u\left(x_{I}, t_{s+1}\right) u^{\prime}\left(x_{I}, t_{s}\right)+$ $u^{\prime}\left(x_{I}, t_{s+1}\right) u\left(x_{I}, t_{s}\right)=u\left(x_{I}, t_{s+1}\right)\left[1-u\left(x_{I}, t_{s+1}\right)\right]$

$$
+u\left(x_{I}, t_{s}\right) u^{\prime}\left(x_{I}, t_{s}\right)
$$

This leads us from the time layer $t_{s}$ to $t_{s+1}$

The wavelet coefficients $a_{s}(i), i=1,2, \ldots ., 2 M$ can be successively calculated from equation (29). This process is started with the initial condition (12). These coefficients are then substituted in equations (25)-(27) to obtain the approximate solutions at different time levels.

\section{Numerical results}

In this section, two examples are considered to check the efficiency and accuracy of the Haar wavelet method. The entire computational work has been done with the help of MATLAB software.

Example 1: Let us consider the Burger-Fisher equation (1), with the initial and boundary conditions in quations (2) and (3) respectively and the exact solution given in equation (4).

Table (1) shows the approximate solution using the proposed method against the exact solution when $\alpha=$ $0.01, \beta=0.01$, and $\gamma=1$, whereas Table (2) shows a comparison between the absolute error of the solution by the proposed method and the absolute error by using the variational iteration method [15] for different values of $x$ and $t$ when $\alpha=0.001, \beta=0.001$, and $\gamma=1$. 
Table (1)

\begin{tabular}{|c|c|c|c|c|}
\hline xi & $\mathrm{ti}$ & Exact solution & Haar solution & Absolute Error \\
\hline 0.01 & 0.02 & 0.500037624999929 & 0.499132084264304 & 0.000905540735625320 \\
\hline 0.02 & & 0.500025124999979 & 0.502892009223431 & 0.002866884223452 \\
\hline 0.03 & & 0.500012624999997 & 0.506445479270577 & 0.006432854270580 \\
\hline 0.04 & & 0.500000125000000 & 0.509811685266022 & 0.009811560266022 \\
\hline 0.05 & & 0.499987625000003 & 0.513007881958884 & 0.013020256958881 \\
\hline 0.01 & 0.03 & 0.500062687499672 & 0.498439354576423 & 0.001623332923248 \\
\hline 0.02 & & 0.500050187499831 & 0.502378619998906 & 0.002328432499075 \\
\hline 0.03 & & 0.500037687499929 & 0.506101165599605 & 0.006063478099676 \\
\hline 0.04 & & 0.500025187499979 & 0.509627059589430 & 0.009601872089451 \\
\hline 0.05 & & 0.500012687499997 & 0.512974341469135 & 0.012961653969137 \\
\hline 0.01 & 0.04 & 0.500087749999099 & 0.497075433069911 & 0.003012316929188 \\
\hline 0.02 & & 0.500075249999432 & 0.501141883179817 & 0.001066633180385 \\
\hline 0.03 & & 0.500062749999671 & 0.50498498853876 & 0.004921748854205 \\
\hline 0.04 & & 0.500050249999831 & 0.508623956709160 & 0.008573706709329 \\
\hline 0.05 & & 0.500037749999928 & 0.512078840448603 & 0.012041090448675 \\
\hline 0.01 & 0.05 & 0.500112812498086 & 0.495074644318046 & 0.005038168180040 \\
\hline 0.02 & & 0.500100312498654 & 0.499212224708285 & 0.000888087790369 \\
\hline 0.03 & & 0.500087812499097 & 0.503122230345461 & 0.003034417846364 \\
\hline 0.04 & & 0.500075312499430 & 0.506825658317006 & 0.006750345817576 \\
\hline 0.05 & & 0.500062812499670 & 0.510341379000510 & 0.010278566500841 \\
\hline 0.01 & 0.06 & 0.500137874996505 & 0.492492007972452 & 0.007645867024053 \\
\hline 0.02 & & 0.500125374997372 & 0.496642393806129 & 0.003482981191243 \\
\hline 0.03 & & 0.500112874998083 & 0.500564964433619 & 0.000452089435537 \\
\hline 0.04 & & 0.500100374998652 & 0.504280738884366 & 0.004180363885715 \\
\hline 0.05 & & 0.500087874999095 & 0.507808607216788 & 0.007720732217693 \\
\hline 0.01 & 0.07 & 0.500162937494232 & 0.489399727599114 & 0.010763209895119 \\
\hline 0.02 & & 0.500150437495461 & 0.493504097865546 & 0.006646339629915 \\
\hline 0.03 & & 0.500137937496501 & 0.497383931385718 & 0.002754006110783 \\
\hline 0.04 & & 0.500125437497386 & 0.501059969090885 & 0.000934531593517 \\
\hline 0.05 & & 0.500112937498079 & 0.504550852381356 & 0.004437914883276 \\
\hline 0.01 & 0.08 & 0.500187999991140 & 0.485882659102875 & 0.014305340888266 \\
\hline 0.02 & & 0.500175499992793 & 0.489883504232251 & 0.010291995760542 \\
\hline 0.03 & & 0.500162999994226 & 0.493666521190520 & 0.006496478803706 \\
\hline 0.04 & & 0.500150499995455 & 0.497251880445489 & 0.002898619549966 \\
\hline 0.05 & & 0.500137999996496 & 0.500657713270939 & 0.000519713274444 \\
\hline
\end{tabular}

Table: 2

\begin{tabular}{|c|c|c|c|c|c|}
\hline $\mathrm{X}$ & $\mathrm{T}$ & $\begin{array}{c}\text { Exact } \\
\text { Sol. }\end{array}$ & $\begin{array}{c}\text { Haar } \\
\text { Sol. }\end{array}$ & $\begin{array}{c}\text { Haar } \\
\text { error }\end{array}$ & $\begin{array}{c}\text { VIM } \\
\text { error }\end{array}$ \\
\hline 0.01 & 0.02 & 0.500015019999996 & 0.499132084264304 & 0.000882935735692 & 0.0025031102 \\
\hline & 0.04 & 0.500035039999943 & 0.498697734403682 & 0.001337305596261 & 0.0025081138 \\
\hline & 0.06 & 0.50005509999777 & 0.498312881454306 & 0.001742218543464 & 0.0025131170 \\
\hline & 0.08 & 0.500075079999436 & 0.497977989064286 & 0.00209709093515 & 0.0025181206 \\
\hline 0.04 & 0.02 & 0.500000020000000 & 0.509811685266022 & 0.009811665266022 & 0.0099961959 \\
\hline & 0.04 & 0.500020039999989 & 0.509360739108795 & 0.009340699108806 & 0.0100011899 \\
\hline & 0.06 & 0.500040059999914 & 0.508960366604204 & 0.00892030660429 & 0.0100061907 \\
\hline & 0.08 & 0.500060079999711 & 0.508611049610424 & 0.008550969610713 & 0.0100111915 \\
\hline
\end{tabular}

\section{Conclusion}

The Burger-Fisher equation has been analyzed using the Haar wavelet method, and the results of this method have been compared with the variational iteration method. The proposed method shows that it is in good agreement with the exact solution and it is a better than variational iteration method.

\section{References}

[1]. Mittal RC, Jiwar R (2009) Differential Quadrature Method for Two Dimensional Burgers' Equations. International Journal of Computational Methods in Engineering Science and Mechanics 10: 450-459.

[2]. Mittal RC, Tripathi A (2014) Numerical solutions of generalized Burgers-Fisher and generalized Burgers- Huxley equations using collocation of cubic B-splines.International Journal of Computer Mathematics http://dx.doi. org/10. 1080/00207160.2014.920834.

[3]. Kheiri H, Ebadi DG (2010) Application of the (G0/G)-expansion method for the Burgers, Fisher, and Burgers- Fisher equations, Acta Universitatis Apulensis 24: 35-44.

[4]. Nawaz R, Ullah H, Islam S, Idrees M (2013) Application of optimal homotopy asymptotic method to Burger equations. Journal of Applied Mathematics http://dx.doi.org/10.1155/2013/387478 doi: 10.1155/ 2013/935154 PMID: 24415902

[5]. J. Lu, G. Yu-Cui, X. Shu-Jiang, "Some new exact solutions to the Burger-Fisher equation and generalized Burgers-Fisher equation", Chinese Physics, vol. 16, no. 9, pp. 1009-1963, 2007.

[6]. X. Wang, Y. Lu, "Exact Solutions of The Extended Burger-Fisher Equation”, Chinese Physics Letters, vol. 7, no. 4, pp. 145, August 1990. 
[7]. Behzadi SS (2011) Numerical solution for solving Burger's-Fisher equation by iterative methods. Mathematical and Computational Applications 6(2): 443-455.

[8]. Nawaz R, Ullah H, Islam S, Idrees M (2013) Application of optimal homotopy asymptotic method to Burger equations. Journal of Applied Mathematics http://dx.doi.org/10.1155/2013/387478 doi: 10.1155/ 2013/935154 PMID: 24415902

[9]. Ismail HNA, Raslan K, Abd Rabboh AA (2004) Adomian decomposition method for Burger's-Huxley and Burger's - Fisher equations. Applied Mathematics and Computation 159: 291-301.

[10]. M. M. Rashidi, D.D. Ganji, S. Dinarvand, "Explicit Analytical Solutions of the Generalized Burger and Burger-Fisher Equations by Homotopy Perturbation Method”, Numerical Methods for Partial Differential Equations, vol. 25, no. 2, pp. 409-417, March 2009.

[11]. Moghimi M, Hejazi FSA (2007) Variational iteration method for solving generalized Burger-Fisher and Burger equations. Chaos, Solitons and Fractals 33: 1756-1761.

[12]. Sari M (2011) Differential quadrature solutions of the generalized Burgers-Fisher equation with a strong stability preserving highorder time integration. Mathematical and Computational Applications 16(2): 477-486.

[13]. Lepik, U., 2007. "Numerical Solution of Evolution Equations" by the Haar Wavelet Method. Appl. Math. and Comput., 185, 695704.

[14]. Hariharan, G., 2013. "An Overview of Haar Wavelet Method for Solving Differential and Integral Equations," World Applied Sciences Journal, 23, 01-14

[15]. Sh.S. Behzadi, M.A.F. Araghi, "Numerical Solution for Solving Burger's-Fisher Eguation by Using Iterative Methods", Mathematical and Computational Applications.

[16]. X.Y.Wang and Z.S.Zhu and Y.K.Lu, Solitary wave solutions of the generalized Burger-Huxley equation, J.Phys. A: Math.Gen 23s $271-274,1990$

[17]. M.J.Lighthill, Viscosity effects in sound waves of finite amplitude. Surveys in Mechanics, Cambridge University Press, 1956

[18]. J.M.Burger, A mathematical model illustrating the theory of turbulence, Advances in Applied Mechanics 1, 171 - 199,1948.

[19]. R.S. Sumana, L.N. Achala, A short report on different wavelets and their structures, Int. J. Res. Engg. Sci. 4(2) (2016) 31-35.

[20]. U. Lepik, H. Hein, Haar wavelets with applications, Springer, 2014.

[21]. R.E. Bellman, R.E. Kalaba, Quasilinearization and nonlinear boundary value problems, American Elsevier Pub. Co., 1965. 